\title{
Evaluation of the 'Jumping to conclusions' bias in different subgroups of the at-risk mental state: from cognitive basic symptoms to UHR criteria
}

\author{
F. Rausch ${ }^{1 *}$, S. Eisenacher ${ }^{1}$, H. Elkin ${ }^{1}$, S. Englisch ${ }^{1}$, S. Kayser ${ }^{2}$, N. Striepens ${ }^{2}$, M. Lautenschlager ${ }^{3}$, \\ A. Heinz ${ }^{3}$, Y. Gudlowski ${ }^{3}$, B. Janssen ${ }^{4}$, W. Gaebel ${ }^{4}$, T. M. Michel ${ }^{5}$, F. Schneider ${ }^{5}$, M. Lambert ${ }^{6}$, \\ D. Naber ${ }^{6}$, G. Juckel ${ }^{7}$, S. Krueger-Oezguerdal ${ }^{7}$, T. Wobrock ${ }^{8}$, A. Hasan', M. Riedel ${ }^{9}$, S. Moritz ${ }^{6}$, \\ H. Müller ${ }^{10}$, J. Klosterkötter ${ }^{10}$, A. Bechdolf ${ }^{10}$, M. Zink ${ }^{1}+$ and M. Wagner ${ }^{2} \dagger$ \\ ${ }^{1}$ Central Institute of Mental Health, Medical Faculty Mannheim, Heidelberg University, Germany; ${ }^{2}$ Department of Psychiatry and Psychotherapy, \\ University of Bonn, Germany; ${ }^{3}$ Department of Psychiatry and Psychotherapy, Charité University Medicine Campus Mitte, Berlin, Germany; \\ ${ }^{4}$ Department of Psychiatry and Psychotherapy, Heinrich-Heine-University Duesseldorf, Germany; ${ }^{5}$ Department of Psychiatry, Psychotherapy and \\ Psychosomatics, University Aachen, Germany; ${ }^{6}$ Department for Psychiatry and Psychotherapy, University Medical Center Hamburg-Eppendorf, \\ Germany; ${ }^{7}$ Department of Psychiatry, Psychotherapy, and Preventive Medicine, Ruhr University Bochum, Germany; ${ }^{8}$ Department of Psychiatry and \\ Psychotherapy, Georg-August-University Goettingen, Goettingen, Germany; ${ }^{9}$ Department of Psychiatry and Psychotherapy, Ludwig-Maximilians- \\ University, Munich, Germany; ${ }^{10}$ Department of Psychiatry and Psychotherapy, University of Cologne, Germany
}

Background. Patients with psychosis display the so-called 'Jumping to Conclusions' bias (JTC) - a tendency for hasty decision-making in probabilistic reasoning tasks. So far, only a few studies have evaluated the JTC bias in 'at-risk mental state' (ARMS) patients, specifically in ARMS samples fulfilling 'ultra-high risk' (UHR) criteria, thus not allowing for comparisons between different ARMS subgroups.

Method. In the framework of the PREVENT (secondary prevention of schizophrenia) study, a JTC task was applied to 188 patients either fulfilling UHR criteria or presenting with cognitive basic symptoms (BS). Similar data were available for 30 healthy control participants matched for age, gender, education and premorbid verbal intelligence. ARMS patients were identified by the Structured Interview for Prodromal Symptoms (SIPS) and the Schizophrenia Proneness Instrument - Adult Version (SPI-A).

Results. The mean number of draws to decision (DTD) significantly differed between ARM -subgroups: UHR patients made significantly less draws to make a decision than ARMS patients with only cognitive BS. Furthermore, UHR patients tended to fulfil behavioural criteria for JTC more often than BS patients. In a secondary analysis, ARMS patients were much hastier in their decision-making than controls. In patients, DTD was moderately associated with positive and negative symptoms as well as disorganization and excitement.

Conclusions. Our data indicate an enhanced JTC bias in the UHR group compared to ARMS patients with only cognitive BS. This underscores the importance of reasoning deficits within cognitive theories of the developing psychosis. Interactions with the liability to psychotic transitions and therapeutic interventions should be unravelled in longitudinal studies.

Received 24 June 2015; Revised 5 February 2016; Accepted 5 February 2016; First published online 20 April 2016

Key words: At-risk mental state, jumping-to-conclusion, metacognition, probabilistic reasoning, prodrome, schizophrenia.

\section{Introduction}

Patients with schizophrenia spectrum disorders display cognitive biases. They present a decreased competence to control their own cognition ('thinking about

\footnotetext{
* Address for correspondence: Dr F. Rausch, Department of Psychiatry and Psychotherapy, Central Institute of Mental Health, Medical Faculty Mannheim/Heidelberg University, Square J5, D-68159 Mannheim, Germany.

(Email: franziska.rausch@zi-mannheim.de)

t These authors contributed equally as joint senior authors.
}

one's thinking'). Typically, impairments affect the abilities to appraise and weigh information effectively, to select appropriate responses including decisions based on perceptions, to cope with cognitive limitations and to build up mental states (Lysaker et al. 2008, 2013). One major cognitive bias pertains to decision-making, the executive part of meta-cognition comprising meta-cognitive monitoring and selfregulation (Flavell et al. 1993). In marked contrast to self-report data indicating that schizophrenia patients consider themselves as rather hesitant and insecure (Freeman et al. 2006), they display an objective 
'Jumping to Conclusions' bias (JTC) - a tendency to hasty decision-making during probabilistic reasoning. The JTC bias in schizophrenia patients was initially described by Hemsley \& Garety (1986). Patients display a premature acceptance of beliefs as true, even when there is limited supporting evidence (Garety \& Freeman, 1999; Fine et al. 2007; Ziegler et al. 2008; Lincoln et al. 2010). JTC is commonly assessed by the 'beads task' (BT; Huq et al. 1988) or slightly modified versions using other stimuli (e.g. fish; Moritz et al. 2013). The task requests a decision after a variable amount of stimuli. Incorporating only one (or two) stimuli to come to a decision is defined as JTC (Garety et al. 1991; Moritz \& Woodward, 2005; Van Dael et al. 2006; Speechley et al. 2010b). Furthermore, in their review that included more than 200 studies Garety and Freeman found JTC to be confirmed as a characteristic of individuals with delusions (Garety \& Freeman, 2013).

Currently, several concepts try to link the JTC bias with general cognitive theories in schizophrenia. For example, JTC and altered salience attribution are supposed to be based on a common cognitive bias (Kapur, 2003; Rubio et al. 2011; Esslinger et al. 2013): A complex dopaminergic dysfunction (Fusar-Poli \& Meyer-Lindenberg, 2012a, b; Howes et al. 2012) is considered to lead to the attribution of aberrant salience to stimuli, which could explain why patients are prone to assuming their hypothesis as confirmed by current evidence and making hasty decisions (Hemsley, 2005; Speechley et al. 2010b). Other authors suggest JTC to be linked to disturbed reward anticipation and learning (Heinz \& Schlagenhauf, 2010; Murray, 2011; Juckel et al. 2012) as well as to altered prediction error signalling assuming a disturbed error-dependent updating of inferences and beliefs about the world (Hemsley \& Garety, 1986; Bentall et al. 2009; Fletcher \& Frith, 2009; Speechley et al. 2010b; Murray, 2011; So et al. 2012).

So far, several functional magnetic resonance imaging (fMRI) studies have defined neural correlates underlying probabilistic reasoning in healthy volunteers and schizophrenia patients, predominantly involving a fronto-striatal-thalamic network (Blackwood et al. 2004; Grinband et al. 2006; Weickert et al. 2009; Furl \& Averbeck, 2011; Koch et al. 2011; Bach \& Dolan, 2012; Morris et al. 2012).

Recently, a hypo-activation in the ventral tegmental area (VTA) and the right ventral striatum (VS) during the point of decision-making was found in schizophrenia patients compared with healthy controls, while a broad cortical activation pattern became apparent during the entire process of probabilistic reasoning (Rausch et al. 2014). A comparably reduced activation pattern within the right VS was also revealed in patients with an 'at-risk mental state' (ARMS; Rausch et al. 2015), which illustrates that underlying neurobiological alterations are already present in the ARMS population. Pathogenetic studies in schizophrenia are limited by illness- and treatment-related confounds, which is why comprehensive investigations of ARMS patients are an extraordinarily useful tool to gain insight into the development of pathology over time.

ARMS patients are commonly identified using cognitive basic symptoms (BS) or 'ultra-high-risk' (UHR) criteria. Patients fulfilling UHR criteria present with attenuated psychotic symptoms (APS) and/or brief limited intermittent psychotic symptoms (BLIPS) or display a genetic risk and a deterioration syndrome (Fusar-Poli et al. 2013).On average, about 22\% of ARMS patients convert to psychosis later on (McGorry et al. 2009; Ruhrmann et al. 2010; Fusar-Poli et al. 2012).

So far, the associations between JTC and neurocognitive properties have been studied in patients with schizophrenia spectrum disorder (Bentall et al. 2009; Garety et al. 2014), in patients with first-episode psychosis (Falcone et al. 2014), in patients with current and remitted delusions (Dudley et al. 1997; Colbert et al. 2010), in delusion-prone individuals (Colbert \& Peters, 2002; White \& Mansell, 2009) and in ARMS patients (Broome et al. 2007). As meta-cognitive impairments are associated with the development of delusions (Fletcher \& Frith, 2009; Speechley et al. 2010a; Moritz et al. 2014; Ross et al. 2015), it seems reasonable that the majority of studies assessed JTC in ARMS samples with upcoming psychotic positive symptoms according to the UHR criteria. However, the ARMS sample can be comprehensively characterized if cognitive BS as well as UHR criteria are assessed using sensitive instruments, such as the Early Recognition Inventory based on IRAOS (ERIraos; Häfner et al. 2012; Rausch et al. 2013; Maurer et al. 2015), or the Schizophrenia Proneness Instrument - Adult Version (SPI-A; Schultze-Lutter et al. 2007) combined with the Structured Interview for Prodromal Symptoms (SIPS; Miller et al. 2003), or the Comprehensive Assessment of At-Risk Mental States (CAARMS; Yung et al. 2005). So far, no data are available on JTC in ARMS stages presenting with cognitive BS only and potential differences compared to ARMS stages fulfilling UHR criteria.

Therefore, we evaluated the baseline data of the secondary prevention of schizophrenia study (PREVENT; Bechdolf et al. 2011). Within this study, several ARMS subgroups were comprehensively characterized for cognitive BS and UHR criteria, and the JTC bias was assessed. We hypothesized we would find a more pronounced JTC bias in ARMS patients fulfilling UHR criteria (ARMS-UHR) in contrast to ARMS patients only 
presenting with cognitive BS (ARMS-BS). Secondary endpoints were the comparison of the ARMS group and a group of healthy control participants that were assessed in a parallel study, as well as correlations of the JTC severity with psychometric data.

\section{Method and materials}

The protocol of the clinical multi-centre PREVENT study (registry identifier: ISRCTN: 02658871) was approved by the respective institutional ethical committees of the trial sites. All participants were provided with detailed information about the study, and written informed consent was obtained prior to study entry. Detailed descriptions of design and setting have been published separately (Bechdolf et al. 2011), but the most important characteristics are summarized below.

\section{Setting and subjects}

The ongoing interventional trial PREVENT was conducted at 12 German Early Intervention Centres (Aachen, Berlin, Bochum, Bonn, Cologne, Dresden, Düsseldorf, Göttingen, Hamburg, Heidelberg, Mannheim, Munich). First, subjects were screened by an Inclusion Criteria Checklist (ICC). For the detailed assessment of UHR criteria, the SIPS including the Scale of Prodromal Symptoms (SOPS) was applied (Miller et al. 2003). Additionally, cognitive BS were assessed by the SPI-A (Schultze-Lutter et al. 2007).

Inclusion criteria for the ARMS group. Age between 18 and 49 years and attribution to one of the following groups: (A) attenuated positive symptoms - presence of at least one of the following symptoms (SOPS scores 3-5): unusual thought content/delusional ideas, suspiciousness/persecutory ideas, grandiosity, perceptual abnormalities/hallucinations, disorganized communication. (B) Brief limited intermittent psychotic symptoms - presence of at least one of the following symptoms: $\leqslant 7$ days resolving spontaneously (SOPS score $=6$ ): hallucinations, delusions, formal thought disorder. (C) Predictive basic symptoms - presence of at least two of the following nine symptoms $(\mathrm{SPI}-\mathrm{A} \geqslant 3)$ at least three times a week during the last 3 months: inability to divide attention, thought interferences, thought pressure, thought blockages, disturbance of receptive speech, disturbance of expressive speech, disturbance in abstract thinking, unstable ideas of reference, captivation of attention by details of the visual field. (D) Family risk plus reduced functioning: any DSM-IV psychotic disorder in first-degree relatives or DSM-IV schizotypal personality disorder of the index person plus impaired global functioning [a 30\% drop in the Global Assessment of Functioning
Scale (GAF) compared to the premorbid level or a score of $\leqslant 50$ during the last 12 months].

Exclusion criteria for the ARMS group. (A) Prior or present antipsychotic treatment for $>1$ week, (B) prior psychotic episode for $>1$ week, (C) present suicidality or self-harming behaviour, (D) alcohol or substance dependence, $(\mathrm{E})$ presence of an organic brain disease, $(\mathrm{F})$ intelligence quotient (IQ) $<70$, (G) contemporary or planned pregnancy, breastfeeding or missing reliable method of contraception in case of sexual activity.

Healthy control participants. Thirty healthy control participants were recruited in parallel in the Mannheim study centre. Control subjects were matched for age, gender, level of education and premorbid verbal intelligence. Prior to study entry, all of the participants were comprehensively evaluated to exclude any positive family history of schizophrenia, bipolar disorder or suicide in first-degree relatives, any previous or current psychiatric disorders according to the Mini-International Neuropsychiatric Interview (M.I.N.I.) and any former or present psychopharmacological treatment.

\section{Methods of cross-sectional assessments}

Socio-demographic parameters such as age, gender, educational level and estimated level of premorbid verbal intelligence [Multiple-Choice Vocabulary Intelligence Test, version B (MWT-B)] were assessed. For psychometric ratings, the SIPS, the SPI-A, the Positive and Negative Syndrome Scale (PANSS), the Montgomery-Asberg Depression Scale (MADRS), the Social and Occupational Functioning Assessment Scale (SOFAS), and the Clinical Global Impression Scale (CGI) were applied. Furthermore, to assess lifetime diagnoses of co-morbid disorders, the Structured Clinical Interviews for DSM-IV, SCID-I and SCID-II were performed at baseline. Additionally, several neurocognitive and metacognitive tests including a JTC task to assess a tendency towards hasty decision-making during probabilistic reasoning were applied. The JTC task was conducted on a PC screen and requested a probabilistic decision after a variable amount of stimuli. Participants successively viewed a total of ten fish in two different colours being fished out of a lake and had to decide which of two possible lakes they were coming from. Colour ratios in the lakes were $80 / 20 \%$ or $20 / 80 \%$, respectively. The coloured fish were presented in a predefined fashion (1-1-1-2-1-1-1-1-2-1). After each fish, subjects were asked to estimate the probability of the fish being taken from lake $\mathrm{A}$ or lake B. Afterwards they were asked if this probability was already sufficient for them to decide for one of the 
two lakes. This task was not repeated, but consisted of a single run (Moritz et al. 2013). It has been developed based on the classical BT (Huq et al. 1988), where subjects viewed beads of two colours being drawn out of a jar and had to decide which of two jars they were drawn from. Further instructions are identical. The ARMS patients underwent the fish task within the PREVENT study whereas the healthy control participants who allowed secondary analyses completed the classical BT and preponderantly also a modified JTC fish task within fMRI scanning, which was part of another study (also see limitations section). Both tasks are detailed described elsewhere (Esslinger et al. 2013; Rausch et al. 2015).

\section{Primary outcome}

The primary endpoint of this cross-sectional investigation was the comparison of the 'draws to decision' (DTD; number of fish needed for a decision) between the different ARMS subgroups either presenting cognitive BS or fulfilling UHR criteria. The UHR group contains a subgroup presenting with attenuated psychotic symptoms (ARMS-APS) and a subgroup presenting with brief limited intermittent psychotic symptoms (ARMS-BLIPS) (solely or additionally to APS). We hypothesized we would find significantly less DTD in the UHR group. Additionally, we compared the number of subjects showing JTC (defined as one or two draws) expecting to find more JTC in the UHR group.

Within the PREVENT study, patients with genetic risk for psychosis in parallel to reduced functioning were classified as 'vulnerability group' and separated from the ARMS subgroups either presenting cognitive BS or fulfilling UHR criteria. We therefore excluded them from our primary analysis, but not from secondary evaluations.

\section{Secondary outcomes}

In order to examine the occurrence of the JTC bias in our ARMS sample per se we compared the total ARMS group with a group of healthy control participants who had been investigated in a parallel project in the Mannheim study centre regarding DTD, as a secondary outcome (Rausch et al. 2015). Again, we evaluated the number of subjects showing JTC in the different groups.

For exploratory reasons, we further stratified the ARMS group for vulnerability, cognitive BS, APS and BLIPS to evaluate group differences. Moreover, we evaluated possible correlations of DTD with clinical characteristics.

\section{Statistics}

Statistical analyses were performed using the SPSS software (IBM SPSS v. 21.0, IBM Corp., USA). Socio-demographic characteristics as well as the primary endpoint were assessed using Student's $t$ tests. Secondary endpoints were evaluated using analysis of variance (ANOVA), two-sided Student's $t$ test and Fisher's exact test to investigate group-specific differences. Correlations were expressed by Pearson's correlation coefficient.

\section{Results}

In the framework of the PREVENT study, 234 ARMS patients were recruited and 188 subjects could be included in the final data analysis. In parallel, a sample of 30 healthy control participants was characterized and could be included in the final data analysis. ARMS patients were identified using SIPS and SPI-A. The mean SIPS sum score was 29.2, the mean SPI-A sum score was 60.54. A total of 42 patients were allocated to ARMS-BS and 132 patients were attributed to ARMS-UHR, presenting APS and/or BLIPS (Table 1). Furthermore, 14 subjects were attributed to the vulnerability group (see Method section, inclusion criteria, group D). There were no significant differences regarding age, gender, education and premorbid verbal intelligence between ARMS-BS and ARMS-UHR (Table 1).

\section{Comparison of the ARMS subgroups (ARMS-BS v. ARMS-UHR)}

As related to the primary endpoint of this study, the DTD significantly differed between ARMS-BS (3.66 \pm 2.35) and ARMS-UHR $(2.62 \pm 2.25)$ as UHR patients needed fewer fish to make a decision $(T=2.30$, $\mathrm{df}=$ $172, p=0.023, d=0.41$; see Fig. 1 ). The mean level of certainty (\%) at the point of decision did not differ between the ARMS subgroups (ARMS-BS: $84.77 \pm 16.62$; ARMS-UHR: $84.10 \pm 13.39 ; T=0.26, d f=152, p=0.799$, $d=0.05)$.

Furthermore, ARMS-UHR tended to fulfil behavioural criteria for JTC (43.2\%) more often than ARMS-BS (26.2\%) (Fisher's exact test, $p=0.069, d=$ 0.28).

\section{Comparison with healthy control participants}

The PREVENT sample of 188 ARMS patients was compared within a secondary analysis with healthy control subjects $(N=30)$ who did not differ significantly regarding age $(p=0.429)$, gender $(p=0.313)$, education as measured by the number of school years $(p=$ 0.232) and estimated premorbid verbal intelligence 
Table 1. Socio-demographic and psychopathological characteristics of ARMS-BS and ARMS-UHR subgroups

\begin{tabular}{llll}
\hline & ARMS-BS $(N=42)$ & ARMS-UHR $(N=132)$ & Comparison \\
\hline Socio-demographics, age, years & $24.10 \pm 3.92$ & $24.77 \pm 5.72$ & $p=0.389$ \\
Gender (female/male) & $14 / 28$ & $48 / 84$ & Fisher: $p=0.854$ \\
School years & $11.24 \pm 1.98$ & $11.21 \pm 2.20$ & $p=0.942$ \\
Multiple choice word test version B (MWT-B) & $27.02 \pm 4.79$ & $27.14 \pm 5.05$ & $p=0.896$ \\
Estimated verbal IQ & $102.64 \pm 12.58$ & $103.60 \pm 13.18$ & $p=0.681$ \\
SIPS & $29.53 \pm 11.92$ & $29.90 \pm 12.50$ & $p=0.872$ \\
SPI-A & $67.18 \pm 32.12$ & $60.91 \pm 35.34$ & $p=0.335$ \\
PANSS & & & $p=0.731$ \\
Total score & $47.50 \pm 10.78$ & $48.26 \pm 12.00$ & $p=0.004^{*}$ \\
Positive symptoms & $9.58 \pm 2.14$ & $11.12 \pm 2.99$ & $p=0.622$ \\
Negative symptoms & $11.37 \pm 4.74$ & $10.95 \pm 4.48$ & $p=0.773$ \\
Global psychopathology & $26.55 \pm 6.57$ & $26.19 \pm 6.62$ & $p=0.076$ \\
Additional scales & & & $p=0.180$ \\
$\quad$ MADRS & $21.64 \pm 7.81$ & $19.07 \pm 8.24$ & $p=0.479$ \\
$\quad$ SOFAS & $50.48 \pm 13.93$ & $53.71 \pm 13.45$ & $4.24 \pm 1.01$ \\
$\quad$ CGI-S & $4.11 \pm 0.98$ & & \\
\hline
\end{tabular}

Data is reported as mean \pm standard deviation (S.D.).

ARMS, At-risk mental state; BS, basic symptoms; CGI-S, Clinical Global Impression - Severity subscore; MADRS, Montgomery-Asberg Depression Scale; PANSS, Positive and Negative Syndrome Scale; SIPS, Structured Interview for Prodromal Symptoms; SOFAS, Social and Occupational Functioning Assessment Scale; SPI-A, Schizophrenia Proneness Instrument - Adult Version; UHR, ultra-high risk;

* Significant.

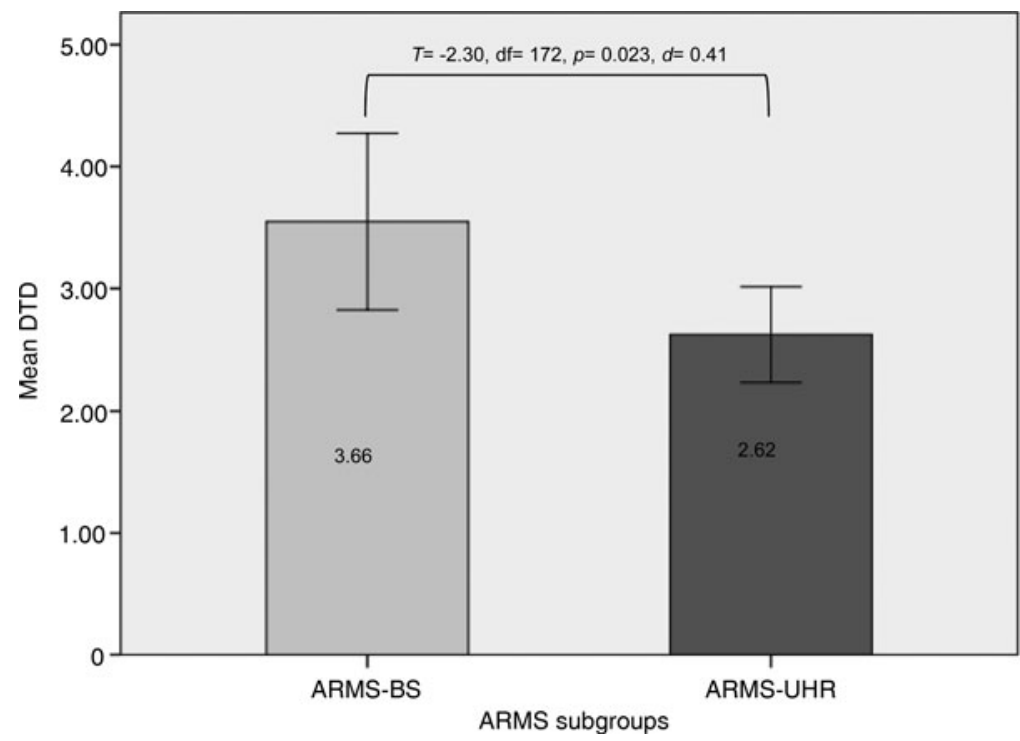

Fig. 1. Displays the mean DTD in the ARMS subgroups. ARMS, At-risk mental state; BS, basic symptoms; DTD, draws to decision; UHR, ultra-high risk.

(MWT-B; $p=0.336$ ). A between-group comparison regarding DTD revealed significant differences, as ARMS patients $(2.90 \pm 2.32)$ needed less stimuli than controls $(4.20 \pm 2.44)$ to make a decision $(T=2.83$, $\mathrm{df}=216, p=0.005, d=0.56$; see Fig. 2). The mean level of certainty (\%) at the point of decision differed significantly between groups, as controls were more secure with their decision (ARMS: 84.64 \pm 13.90 ; controls: $89.67 \pm 8.09 ; T=2.76, \mathrm{df}=64.5, p=0.008, d=0.43$ ).

Moreover, the ARMS group tended to show more JTC (38.8\%) compared to the control group (20.0\%) (Fisher's exact test, $p=0.064, d=0.25$ ). 


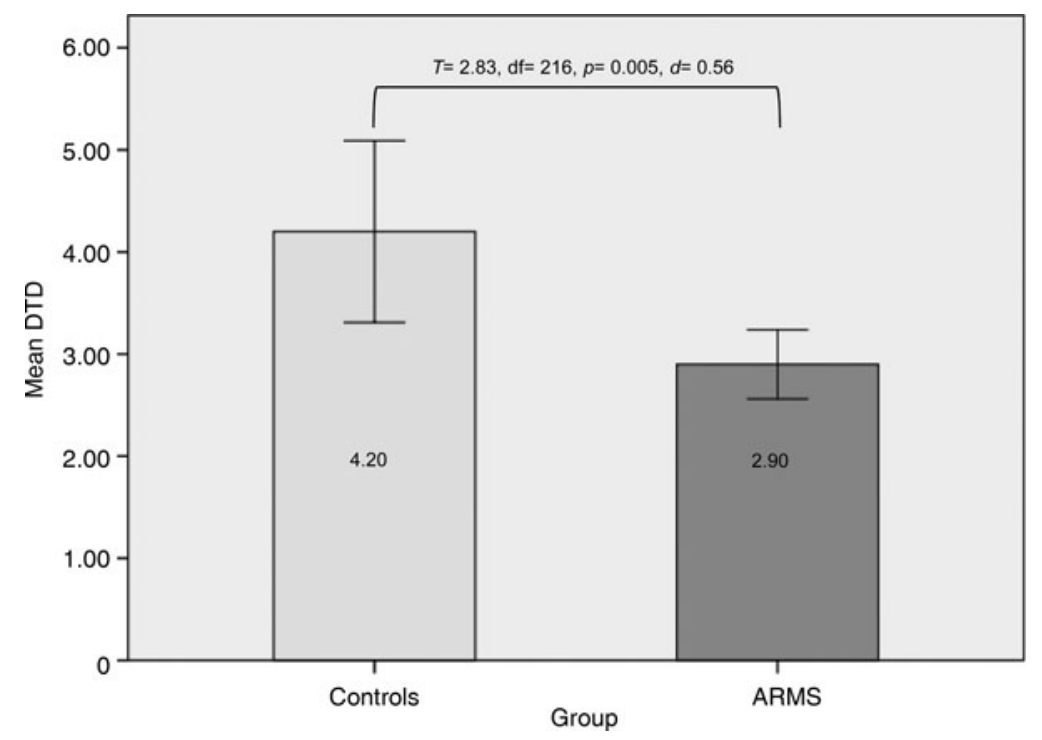

Fig. 2. Displays the mean DTD of ARMS patients and healthy control participants. ARMS, At-risk mental state; DTD, draws to decision.

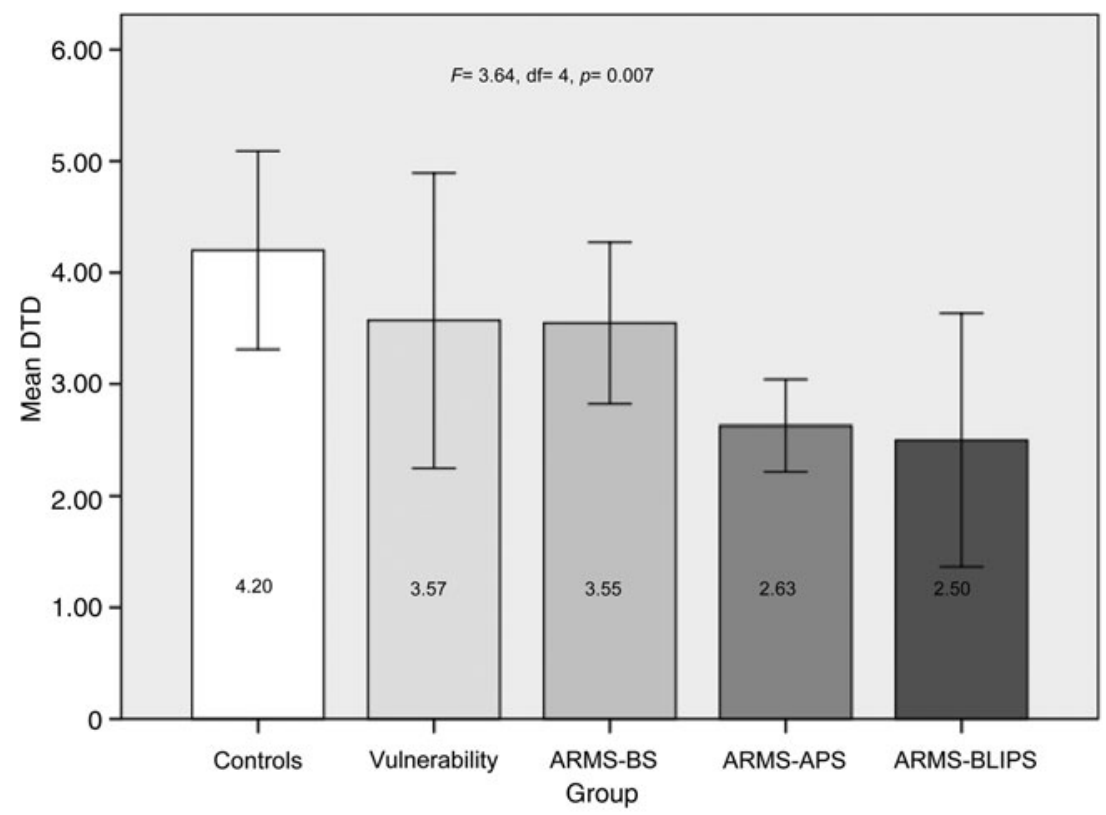

Fig. 3. Displays the mean DTD in the different symptom groups and the healthy control participants. APS, Attenuated psychotic symptoms; ARMS, at-risk mental state; BLIPS, brief limited intermittent psychotic symptoms; BS, basic symptoms; DTD, draws to decision.

\section{Exploratory analysis}

The between-group comparison of the entire model including the vulnerability group, ARMS-BS, ARMSUHR-APS, ARMS-UHR-BLIPS and healthy controls using a one-way ANOVA revealed a significant difference ( $F=3.64, \mathrm{df}=4, p=0.007, d=0.56$ ). Fig. 3 displays the group means of DTD for all subgroups.
Additionally, a prognostic score allowing for an individualized estimation of the transition risk was recently proposed by Ruhrmann et al. (2010) and Müller et al. (unpublished data). The prognostic score was calculated as $(1.571 \times$ SIPS-positive score $>16)+(0.865 \times$ SCID-II score for schizotypal personality disorder $=$ $3)+(0.793 \times$ bizarre thinking score $>2)+(1.037 \times$ sleep 
disturbance score $>2)+[((100-$ highest SOFAS in the past year) -34.64$) \times 0.033]+[$ (years of education recoded -12.52$) \times 0.250$ ]. Stratifying the ARMS group for the corresponding risk classes revealed a decrease of mean DTDs in parallel to an assumed increase of the risk for transition, with DTD $=3.15$ in risk class 1 , 2.79 in risk class 2, 2.73 in risk class 3 and 2.00 in risk class 4 . The between-group comparison including the four risk classes and the healthy control group using a one-way ANOVA revealed a significant difference $(F=2.59, \mathrm{df}=4, p=0.038, d=0.57)$.

Furthermore, we evaluated possible associations of DTD with the early recognition scales SIPS and SPI-A and their subscales, but no significant correlations became apparent. However, in the entire ARMS group the correlation of DTD with clinical characteristics revealed significant correlations with the PANSS total score $(r=-0.248, p=0.001)$ and all scores of the PANSS five-factor model (Van der Gaag et al. 2006) except Emotional Distress that displayed a trend (Positive: $r=-0.228, p=0.003$; Negative: $r=-0.178, p=0.023$; Disorganization: $r=-0.223, \quad p=0.004$; Excitement: $r=-0.199, p=0.011$; Emotional Distress: $r=-0.147, p=$ 0.061; all two-sided). Stratifying the ARMS group for ARMS-UHR and ARMS-BS, similar correlations became apparent in ARMS-UHR, but in ARMS-BS no significant correlations between DTD and PANSS scores were revealed. Regarding MADRS, SOFAS and CGI, no correlations became evident.

Furthermore, in patients the mean level of certainty (\%) at the point of decision significantly correlated with DTD $(r=0.360, p \leqslant 0.001)$, as subjects were more secure when they evaluated more stimuli before making a decision. However, no correlations with clinical characteristics were observed.

As DTD might be affected by the premorbid verbal intelligence (MWT-B) we tested for possible associations in the total sample, but no significant correlations became apparent $(r=0.021, p=0.763)$.

Due to the exploratory character of the correlation study, the levels of statistical significance were reported without correction for multiple testing.

\section{Discussion}

Our findings indicate that ARMS patients fulfilling UHR criteria, in contrast to patients only presenting with cognitive BS, display an increased behavioural propensity to hasty decision-making. Furthermore, the comparison of ARMS patients and healthy control participants revealed that ARMS patients needed fewer stimuli than controls to reach a decision, confirming previous findings (Broome et al. 2007; Rausch et al. 2015).
In comparison with healthy controls, the ARMS patients were more insecure about their decision. This pattern of findings suggests that ARMS patients tend to decide after fewer stimuli and on the basis of less certainty, which corresponds to findings of Moritz and colleagues, pointing to hasty decisionmaking based on low subjective certainty (Moritz et al. 2006) and suggesting a liberal acceptance bias to be responsible for decision-making biases in schizophrenia (Moritz et al. 2007, 2008). Moderating effects of the entire amount of information on the subjective certainty seem possible, as suggested be the observed correlation of DTD and certainty.

To our knowledge, so far there is no study comparing different ARMS subgroups (ARMS-BS and ARMS-UHR) regarding JTC. Our data correspond to the results of studies exclusively assessing UHR samples (Broome et al. 2007). Furthermore, our findings add to data on schizophrenia patients that suggest JTC as a 'state' associated with psychotic symptoms as well as a maintaining factor for delusions (Jolley et al. 2014). On the other hand there are findings that support the theory of JTC as a trait phenomenon (Moritz \& Woodward, 2005; Van Dael et al. 2006; Garety \& Freeman, 2013; Falcone et al. 2014).

However, the JTC bias seems to be an early cognitive marker of the emerging psychotic state. In a recent fMRI investigation, ARMS patients defined according to the ERIraos (Häfner et al. 2012; Rausch et al. 2013; Maurer et al. unpublished data) also presented with JTC and showed reduced activation of the right VS during probabilistic decision-making (Rausch et al. 2015). Our results support associations of JTC with general delusion development, as the ARMS-BLIPS group in average needed the fewest fish to come to a decision. This might indicate a mechanism for the development of BLIPS and disturbed cognition during prodromal states. Taking into account the correlation of DTD with Positive, Negative, Disorganization and Excitement subscores of the PANSS, the JTC bias might be a hint towards general and underlying cognitive alterations that induce the development of psychotic symptoms in general, not only positive symptoms.

Our findings further underpin the proposals of disturbances related to reward anticipation (Heinz \& Schlagenhauf, 2010; Murray, 2011; Juckel et al. 2012) and the ability to propagate prediction errors in a hierarchical Bayesian inference framework between lowerand higher-level systems in schizophrenia patients (Lee \& Mumford, 2003; Fletcher \& Frith, 2009; Friston, 2010; Dura-Bernal et al. 2012), extending them to the ARMS.

Furthermore, since our findings point to a successive increase of JTC during the course of the ARMS, this might suppose the late ARMS stage as a progress - 
not only theoretically, but underpinning the patients' increasing cognitive impairment. This fact is reflected in the primary endpoint of the PREVENT trial, defining both transitions from the early to the late ARMS and to the psychotic state. Thus, JTC might display a measure of progressing functional alterations predominantly assigned to striatal brain regions that is accessible for scientific observation.

Besides JTC, several other measures are supposed to reflect the neurobiology in early psychotic states. For instance, Bodatsch et al. (2011) found a significant reduction in the duration mismatch-negativity in those ARMS patients who later converted to psychosis, compared to those without a transition. These results might contribute to an individualized prediction of the transition risk. The study of Frommann et al. (2011) revealed more pronounced neurocognitive impairments (e.g. in the domains of working memory, processing speed and memory) in an UHR sample compared to patients only presenting with cognitive BS. Similarly, Koutsouleris et al. (2012b) found executive functioning and verbal IQ deficits to be particular properties of the late ARMS stage. Moreover, they suggest MRI-based biomarkers, e.g. alterations in prefrontal perisylvian and subcortical brain structures, to depict a helpful tool for an improved estimation of the psychosis risk (Koutsouleris et al. 2012a).

However, it remains to be seen if a predictive value regarding transitions to psychosis can also be attributed to the JTC bias. Therefore, follow-up investigations within the PREVENT sample are mandatory.

Finally, our results underscore the importance of JTC and meta-cognitive deficits in general within cognitive theories of the developing psychosis as JTC is suggested as a state predominantly co-occurring with UHR stages. On the other hand, one could argue that subjects that are not liable to show JTC perhaps will not develop APS or BLIPS. Moreover, these findings highlight the need for complemented psychosis and ARMS treatments with bias modification programmes like meta-cognitive (Moritz et al. 2014) and reasoning (Ross et al. 2011) training. For instance, Andreou et al. (2014) observed a positive association between an improvement of the JTC bias and the vocational outcome in patients with schizophrenia, also underpinning the importance of interventions regarding meta-cognitive deficits.

\section{Limitations}

This study is limited by its cross-sectional design, thus prohibiting accounts on the inherent transition risk and on differences regarding the transition risk in several ARMS subgroups. Therefore, the analysis of the longitudinal data of the PREVENT study is crucial.
Furthermore, it must be re-emphasized that the healthy control participants were not recruited within PREVENT and underwent the classical BT instead of the fish task as described above. Regarding DTD, the instructions were identical, but outside this construct slight wording-differences were present. It might be discussed, whether the more salient fish paradigm might have enforced a tendency towards JTC behaviour (Dudley et al. 1997; Young \& Bentall, 1997). However, we were able to control for this caveat: a comparison of DTD according to the classical BT (mean $4.20 \pm 2.44$ ) revealed no significant difference to DTD extracted from a JTC fish task that was applied to a subgroup $(n=28)$ of the healthy control participants during fMRI scanning (mean 4.14 \pm 2.29 ). Therefore we propose that it is feasible to draw a robust measure like DTD out of this data, especially because the mean DTD in our control group matches to scores of control groups reported in other studies (Moritz \& Woodward, 2005; White \& Mansell, 2009; Colbert et al. 2010; Garety and Freeman, 2013; Ermakova et al. 2014; Rausch et al. 2014).

\section{Conclusions}

Data found in our study indicate an enhancement of hasty decision-making in patients fulfilling UHR criteria compared to patients presenting with cognitive BS only. Finally, interactions of JTC with the liability of psychotic transitions and therapeutic interventions should be unravelled during the longitudinal phase of PREVENT.

\section{Acknowledgements}

The authors acknowledge the support of this project provided by Judith Gimpel, M.Sc. Psych., Dr Dana Beck, Dipl.-Psych., Prof. Dr. Dr René Hurlemann, NN. The German Research Foundation provided funding to Prof. Dr. J. Klosterkötter (DFG, grant KL 970/7-1) and Prof. Dr. M. Zink (ZI 1253/3-1 and 3-2).

\section{Declaration of Interest}

S. Englisch has received travel expenses and consultant fees from AstraZeneca, Bristol-Myers Squibb, Eli-Lilly, Janssen Cilag, Lundbeck, Otsuka Pharma, Pfizer Pharma, Roche Pharma and Servier. M. Zink has received unrestricted scientific grants from the German Research Foundation (DFG), and Servier; further speaker and travel grants were provided from AstraZeneca, Lilly, Pfizer Pharma GmbH, BristolMyers Squibb Pharmaceuticals, Otsuka, Servier, Lundbeck and Trommsdorff. All other authors report no conflict of interest. 


\section{References}

Andreou C, Treszl A, Roesch-Ely D, Kother U, Veckenstedt R, Moritz S (2014). Investigation of the role of the jumping-to-conclusions bias for short-term functional outcome in schizophrenia. Psychiatry Research 218, 341-347.

Bach DR, Dolan RJ (2012). Knowing how much you don't know: a neural organization of uncertainty estimates. Nature Reviews Neuroscience 13, 572-586.

Bechdolf A, Müller H, Stutzer H, Wagner M, Maier W, Lautenschlager M, Heinz A, Janssen B, Gaebel W, Michel TM, Schneider F, Lambert $M$, Naber $D$, Brüne $M$, Krüger-Özgürdal S, Wobrock T, Riedel M, Klosterkötter J (2011). Rationale and baseline characteristics of PREVENT: a second-generation intervention trial in subjects at-risk (prodromal) of developing first-episode psychosis evaluating cognitive behavior therapy, aripiprazole, and placebo for the prevention of psychosis. Schizophrenia Bulletin 37 (Suppl. 2), S111-S121.

Bentall RP, Rowse G, Shryane N, Kinderman P, Howard R, Blackwood N, Moore R, Corcoran R (2009). The cognitive and affective structure of paranoid delusions: a transdiagnostic investigation of patients with schizophrenia spectrum disorders and depression. Archives of General Psychiatry 66, 236-247.

Blackwood N, Fytche D, Simmons A, Bentall R, Murray R, Howard R (2004). The cerebellum and decision making under uncertainty. Cognitive Brain Research 20, 46-53.

Bodatsch M, Ruhrmann S, Wagner M, Müller R, Schultze-Lutter F, Frommann I, Brinkmeyer J, Gaebel W, Maier W, Klosterkötter J, Brockhaus-Dumke A (2011). Prediction of psychosis by mismatch negativity. Biological Psychiatry 69, 959-966.

Broome MR, Johns LCD, Valli IMD, Wooley JBM, Tabraham PD, Brett C, Valmaggia L, Peteres E, Garety PA, McGuire PKP (2007). Delusion formation and reasoning biases in those at clinical high risk for psychosis. British Journal of Psychiatry 191, s38-s42.

Colbert SM, Peters ER (2002). Need for closure and jumping-to-conclusions in delusion-prone individuals. Journal of Nervous \& Mental Disease 190, 27-31.

Colbert SM, Peters E, Garety P (2010). Jumping to conclusions and perceptions in early psychosis: relationship with delusional beliefs. Cognitive Neuropsychiatry 15, 422-440.

Dudley RE, John CH, Young AW, Over DE (1997). Normal and abnormal reasoning in people with delusions. British Journal of Clinical Psychology 36, 243-258.

Dura-Bernal S, Wennekers T, Denham SL (2012). Top-down feedback in an HMAX-Like cortical model of object perception based on hierarchical bayesian networks and belief propagation. PLOS ONE 7, e48216.

Ermakova AO, Ramachandra P, Corlett PR, Fletcher PC, Murray GK (2014). Effects of methamphetamine administration on information gathering during probabilistic reasoning in healthy humans. PLOS ONE. 9, e102683.

Esslinger C, Braun U, Schirmbeck F, Santos A, Meyer-Lindenberg A, Zink M, Kirsch P (2013). Activation of midbrain and ventral striatal regions implicates salience processing during a modified beads task. PLOS ONE 8, e58536.

Falcone MA, Murray RM, Wiffen BD, O'Connor JA, Russo M, Kolliakou A, Stilo S, Taylor H, Gardner-Sood P, Paparelli A, Jichi F, Di FM, David AS, Freeman D, Jolley S (2014). Jumping to conclusions, neuropsychological functioning, and delusional beliefs in first episode psychosis. Schizophrenia Bulletin. Published online: 22 July 2014. doi:10.1093/schbul/sbu104.

Fine C, Gardner M, Craigie J, Gold I (2007). Hopping, skipping or jumping to conclusions? Clarifying the role of the JTC bias in delusions. Cognitive Neuropsychiatry 12, 46-77.

Flavell JH, Green FL, Flavell ER (1993). Children's understanding of the stream of consciousness. Child Development 64, 387-398.

Fletcher PC, Frith CD (2009). Perceiving is believing: a bayesian approach to explaining the positive symptoms of schizophrenia. Nature Reviews Neuroscience 10, 48-58.

Freeman D, Garety P, Kuipers E, Colbert S, Jolley S, Fowler D, Dunn G, Bebbington P (2006). Delusions and decision-making style: use of the Need for Closure Scale. Behaviour Research and Therapy 44, 1147-1158.

Friston K (2010). The free-energy principle: a unified brain theory? Nature Reviews Neuroscience 11, 127-138.

Frommann I, Pukrop R, Brinkmeyer J, Bechdolf A, Ruhrmann S, Berning J, Decker P, Riedel M, Möller HJ, Wölwer W, Gaebel W, Klosterkötter J, Maier W, Wagner M (2011). Neuropsychological profiles in different at-risk states of psychosis: executive control impairment in the early- and additional memory dysfunction in the late- prodromal state. Schizophrenia Bulletin 37, 861-873.

Furl N, Averbeck BB (2011). Parietal cortex and insula relate to evidence seeking relevant to reward-related decisions. Journal of Neuroscience 31, 17572-17582.

Fusar-Poli P, Bonoldi I, Yung AR, Borgwardt S, Kempton MJ, Valmaggia L, Barale F, Caverzasi E, McGuire P (2012). Predicting psychosis: meta-analysis of transition outcomes in individuals at high clinical risk. Archives of General Psychiatry 69, 220-229.

Fusar-Poli P, Borgwardt S, Bechdolf A, Addington J, Riecher-Roessler A, Schultze-Lutter F, Keshavan M, Wood S, Ruhrmann S, Seidman LJ, Valmaggia L, Cannon T, Velthorst E, de Haan L, Cornblatt B, Bonoldi I, Birchwood M, McGlashan T, Carpenter WT, McGorry PD, Klosterkoetter J, McGuire P, Yung A (2013). The psychosis high-risk state: a comprehensive state-of-the-art review. JAMA Psychiatry 70, 107-120.

Fusar-Poli P, Meyer-Lindenberg A (2012a). Striatal presynaptic dopamine in schizophrenia, part I: meta-analysis of dopamine active transporter (DAT) density. Schizophrenia Bulletin 39, 22-32.

Fusar-Poli P, Meyer-Lindenberg A (2012b). Striatal presynaptic dopamine in schizophrenia, part II: meta-analysis of [18F/11C]-DOPA PET studies. Schizophrenia Bulletin 39, 33-42.

Garety PA, Freeman D (1999). Cognitive approaches to delusions: a critical review of theories and evidence. British Journal of Clinical Psychology 38, 113-154. 
Garety PA, Freeman D (2013). The past and future of delusions research: from the inexplicable to the treatable. British Journal of Psychiatry 203, 327-333.

Garety P, Waller H, Emsley R, Jolley S, Kuipers E, Bebbington P, Dunn G, Fowler D, Hardy A, Freeman D (2014). Cognitive mechanisms of change in delusions: an experimental investigation targeting reasoning to effect change in paranoia. Schizophrenia Bulletin. Published online: 21 July 2014. doi:10.1093/schbul/sbu103.

Garety PA, Hemsley DR, Wessely S (1991). Reasoning in deluded schizophrenic and paranoid patients. Biases in performance on a probabilistic inference task. Journal of Nervous and Mental Diseases 179, 194-201.

Grinband J, Hirsch J, Ferrera VP (2006). A neural representation of categorization uncertainty in the human brain. Neuron 49, 757-763.

Häfner H, Bechdolf A, Klosterkötter J, Maurer K (2012). Psychosen - Früherkennung und Frühintervention. In Der Praxisleitfaden. Schattauer: Stuttgart, pp. 67-87.

Heinz A, Schlagenhauf F (2010). Dopaminergic dysfunction in schizophrenia: salience attribution revisited. Schizophrenia Bulletin 36, 472-485.

Hemsley DR (2005). The schizophrenic experience: taken out of context? Schizophrenia Bulletin 31, 43-53.

Hemsley DR, Garety PA (1986). The formation of maintenance of delusions: a Bayesian analysis. British Journal of Psychiatry 149, 51-56.

Howes OD, Kambeitz J, Kim E, Stahl D, Slifstein M, bi-Dargham A, Kapur S (2012). The nature of dopamine dysfunction in schizophrenia and what this means for treatment: a meta-analysis of imaging studies. Archives of General Psychiatry 69, 776-786.

Huq SF, Garety PA, Hemsley DR (1988). Probabilistic judgements in deluded and non-deluded subjects. Quarterly Journal of Experimental Psychology. A 40, 801-812.

Jolley S, Thompson C, Hurley J, Medin E, Butler L, Bebbington P, Dunn G, Freeman D, Fowler D, Kuipers E, Garety P (2014). Jumping to the wrong conclusions? An investigation of the mechanisms of reasoning errors in delusions. Psychiatry Research 219, 275-282.

Juckel G, Friedel E, Koslowski M, Witthaus H, Özgürdal S, Gudlowski Y, Knutson B, Wrase J, Brüne M, Heinz A, Schlagenhauf F (2012). Ventral striatal activation during reward processing in subjects with ultra-high risk for schizophrenia. Neuropsychobiology 66, 50-56.

Kapur S (2003). Psychosis as a state of aberrant salience: a framework linking biology, phenomenology, and pharmacology in schizophrenia. American Journal of Psychiatry 160, 13-23.

Koch K, Wagner G, Schachtzabel C, Schultz CC, Guellmar D, Reichenbach JR, Sauer H, Schloesser RGM (2011). Neural activation and radial diffusivity in schizophrenia: combined $\mathrm{fMRI}$ and diffusion tensor imaging study. British Journal of Psychiatry 198, 223-229.

Koutsouleris N, Borgwardt S, Meisenzahl EM, Bottlender R, Möller HJ, Riecher-Rössler A (2012a). Disease prediction in the at-risk mental state for psychosis using neuroanatomical biomarkers: results from the FePsy study. Schizophrenia Bulletin 38, 1234-1246.
Koutsouleris N, Davatzikos C, Bottlender R, Patschurek-Kliche K, Scheuerecker J, Decker P, Gaser C, Möller HJ, Meisenzahl EM (2012b). Early recognition and disease prediction in the at-risk mental states for psychosis using neurocognitive pattern classification. Schizophrenia Bulletin 38, 1200-1215.

Lee TS, Mumford D (2003). Hierarchical bayesian inference in the visual cortex. Journal of the Optical Society of America A 20, 1434-1448.

Lincoln TM, Ziegler M, Mehl S, Rief W (2010). The jumping to conclusions bias in delusions: specificity and changeability. Journal of Abnormal Psychology 119, 40-49.

Lysaker PH, Gumley A, Luedtke B, Buck KD, Ringer JM, Olesek K, Kukla M, Leonhardt BL, Popolo R, Dimaggio G (2013). Social cognition and metacognition in schizophrenia: evidence of their independence and linkage with outcomes. Acta Psychiatrica Scandinavica 127, 239-247.

Lysaker PHP, Warman DMP, Dimaggio GM, Procacci MM, LaRocco VAM, Clark LKM, Dike CAM, Nicolo GM (2008). Metacognition in schizophrenia: associations with multiple assessments of executive function. Journal of Nervous \& Mental Disease 196, 384-389.

McGorry PD, Nelson B, Amminger GP, Bechdolf A, Francey SM, Berger G, Riecher-Rössler A, Klosterkötter J, Ruhrmann S, Schultze-Lutter F, Nordentoft M, Hickie I, McGuire P, Berk M, Chen EY, Keshavan MS, Yung AR (2009). Intervention in individuals at ultra high risk for psychosis: a review and future directions. Journal of Clinical Psychiatry 70, 1206-1212.

Miller TJ, McGlashan TH, Rosen JL, Cadenhead K, Ventura J, McFarlane W, Perkins DO, Pearlson GD, Woods SW (2003). Prodromal assessment with the structured interview for prodromal syndromes and the scale of prodromal symptoms: predictive validity, interrater reliability, and training to reliability. Schizophrenia Bulletin 29, 703-715.

Moritz S, Woodward TS (2005). Jumping to conclusions in delusional and non-delusional schizophrenic patients. British Journal of Clinical Psychology 44, 193-207.

Moritz S, Andreou C, Schneider BC, Wittekind CE, Menon M, Balzan RP, Woodward TS (2014). Sowing the seeds of doubt: a narrative review on metacognitive training in schizophrenia. Clinical Psychology Review 34, 358-366.

Moritz S, Veckenstedt R, Bohn F, Hottenrott B, Scheu F, Randjbar S, Aghotor J, Kother U, Woodward TS, Treszl A, Andreou C, Pfueller U, Roesch-Ely D (2013).

Complementary group metacognitive training (MCT) reduces delusional ideation in schizophrenia. Schizophrenia Research 151, 61-69.

Moritz S, Woodward TS, Hausmann D (2006). Incautious reasoning as a pathogenetic factor for the development of psychotic symptoms in schizophrenia. Schizophrenia Bulletin 32, 327-331.

Moritz S, Woodward TS, Jelinek L, Klinge R (2008). Memory and metamemory in schizophrenia: a liberal acceptance account of psychosis. Psychological Medicine 38, 825-832.

Moritz S, Woodward TS, Lambert M (2007). Under what circumstances do patients with schizophrenia jump to conclusions? A liberal acceptance account. British Journal of Clinical Psychology 46, 127-137. 
Morris RW, Vercammen A, Lenroot R, Moore L, Langton JM, Short B, Kulkarni J, Curtis J, O'Donnell M, Weickert CS, Weickert TW (2012). Disambiguating ventral striatum fMRI-related bold signal during reward prediction in schizophrenia. Molecular Psychiatry 17, 280-289.

Murray GK (2011). The emerging biology of delusions. Psychological Medicine 41, 7-13.

Rausch F, Eifler S, Esser A, Esslinger C, Schirmbeck F, Meyer-Lindenberg A, Zink M (2013). The early recognition inventory ERIraos detects at risk mental states of psychosis with high sensitivity. Comprehensive Psychiatry. Published online: 4 June 2013. doi:10.1016/j. comppsych.2013.04.016.

Rausch F, Mier D, Eifler S, Esslinger C, Schilling C, Schirmbeck F, Englisch S, Meyer-Lindenberg A, Kirsch P, Zink M (2014). Reduced activation in ventral striatum and ventral tegmental area during probabilistic decisionmaking in schizophrenia. Schizophrenia Research 156, 143149.

Rausch F, Mier D, Eifler S, Fenske S, Schirmbeck F, Englisch S, Schilling C, Meyer-Lindenberg A, Kirsch P, Zink M (2015). Reduced activation in the ventral striatum during probabilistic decision-making in patients in an at-risk mental state. Journal of Psychiatry and Neuroscience 40, 140191.

Ross K, Freeman D, Dunn G, Garety P (2011). A randomized experimental investigation of reasoning training for people with delusions. Schizophrenia Bulletin 37, 324-333.

Ross RM, McKay R, Coltheart M, Langdon R (2015). Jumping to conclusions about the beads task? a meta-analysis of delusional ideation and data-gathering. Schizophrenia Bulletin. Published online: 22 January 2015. doi:10.1093/schbul/sbu187.

Rubio JL, Ruiz-Veguilla M, Hernandez L, Barrigon ML, Salcedo MD, Moreno JM, Gomez E, Moritz S, Ferrin M (2011). Jumping to conclusions in psychosis: a faulty appraisal. Schizophrenia Research 133, 199-204.

Ruhrmann S, Schultze-Lutter F, Salokangas RK, Heinimaa M, Linszen D, Dingemans P, Birchwood M, Patterson P, Juckel G, Heinz A, Morrison A, Lewis S, von Reventlow HG, Klosterkötter J (2010). Prediction of psychosis in adolescents and young adults at high risk: results from the prospective European prediction of psychosis study. Archives of General Psychiatry 67, 241251.
Schultze-Lutter F, Addington J, Ruhrmann S, Klosterkötter J (2007). Schizophrenia Proneness Instrument - Adult Version. Giovanni Fioriti Editore: Rome, Italy.

So SH, Freeman D, Dunn G, Kapur S, Kuipers E, Bebbington P, Fowler D, Garety PA (2012). Jumping to conclusions, a lack of belief flexibility and delusional conviction in psychosis: a longitudinal investigation of the structure, frequency, and relatedness of reasoning biases. Journal of Abnormal Psychology 121, 129-139.

Speechley WJ, Murray CB, McKay RM, Munz MT, Ngan ETC (2010a). A failure of conflict to modulate dual-stream processing may underlie the formation and maintenance of delusions. European Psychiatry 25, 80-86.

Speechley WJ, Whitman JC, Woodward TS (2010b). The contribution of hypersalience to the 'jumping to conclusions' bias associated with delusions in schizophrenia. Journal of Psychiatry \& Neuroscience 35, 7-17.

Van Dael F, Versmissen D, Janssen I, Myin-Germeys I, van Os J, Krabbendam L (2006). Data gathering: biased in psychosis? Schizophrenia Bulletin 32, 341-351.

Van der Gaag M, Hoffmann T, Remijsen M, Hijman R, de Haan L, van Meijel B, van Harten PN, Valmaggia L, De Hert M, Cuijpers A, Wiersma D (2006). The five-factor model of the Positive and Negative Syndrome Scale II: a ten-fold cross-validation of a revised model. Schizophrenia Research 85, 280-287.

Weickert TW, Goldberg TE, Callicott JH, Chen Q, Apud JA, Das S, Zoltick BJ, Egan MF, Meeter M, Myers C, Gluck MA, Weinberger DR, Mattay VS (2009). Neural correlates of probabilistic category learning in patients with schizophrenia. Journal of Neuroscience 29, 1244-1254.

White LO, Mansell W (2009). Failing to ponder? Delusion-prone individuals rush to conclusions. Clinical Psychology \& Psychotherapy 16, 111-124.

Young HF, Bentall RP (1997). Probabilistic reasoning in deluded, depressed and normal subjects: effects of task difficulty and meaningful versus non-meaningful material. Psychological Medicine 27, 455-465.

Yung A, Yuen HP, McGorry PD, Phillips LJ, Kelly D, Dell'Olio M, Francey SM, Cosgrave EM, Killackey E, Stanford C, Godfrey K, Buckby J (2005). Mapping the onset of psychosis: the comprehensive assessment of at-risk mental states. Australian \& New Zealand Journal of Psychiatry 39, 964-971.

Ziegler M, Rief W, Werner SM, Mehl S, Lincoln TM (2008). Hasty decision-making in a variety of tasks: does it contribute to the development of delusions? Psychology $\mathcal{E}$ Psychotherapy: Theory, Research \& Practice 81, 237-245. 